\title{
Imaging of 3D Tissue-Engineered Models of Oral Cancer Using 890 and 1300 nm opticat Goherence Tomography
}

DOI 10.17691/stm2015.7.1.08

Received December 8, 2014

J. Boadi, MPhys, PhD Student, Department of Materials Science and Engineering';

J. Fernandes, MRes, MRes Graduate, Department of Oncology, Faculty of Medicine, Dentistry and Health2;

s. Mittar, PhD, PDRA, Department of Materials Science and Engineering';

V. Hearnden, PhD, PDRA, Department of Oncology, Faculty of Medicine, Dentistry and Health2;

Z. Lu, PhD, PDRA, Department of Materials Science and Engineering';

Department of Electrical \& Electronic Engineering;

S. MacNeil,PhD, Professor, Department of Materials Science and Engineering ${ }^{1}$;

M.H. Thornhill, PhD, Professor";

๑. Murdoch, PhD, Lecturer4;

K.D. Hunter, PhD, Reader4;

S.J. Matcher, PhD, Reader, Department of Materials Science and Engineering ${ }^{1,5}$

${ }^{1}$ The Kroto Research Institute, University of Sheffield, North Campus, Broad Lane, Sheffield, S3 7HQ,

United Kingdom;

${ }^{2}$ Medical School, University of Sheffield, Beech Hill Road, Sheffield, S10 2RX, United Kingdom;

${ }^{3}$ Nanoscience \& Technology Centre, University of Sheffield, North Campus, Broad Lane, Sheffield, S3 7HQ,

United Kingdom;

${ }^{4}$ School of Clinical Dentistry, University of Sheffield, Claremont Crescent, Sheffield, S10 2TA, United Kingdom;

${ }^{5}$ Insigneo Institute for in silico Medicine: F Floor- Room F19, The Pam Liversidge Building, Sir Frederick Mappin Building,

The University of Sheffield, Mappin Street, Sheffield, S1 3JD, United Kingdom

Optical coherence tomography (OCT) generates its primary form of contrast from elastic backscatter. It is now the gold standard technique for retinal screening and is emerging rapidly in cardiovascular research however it remains a research goal to establish it to the same degree in epithelial cancer detection and diagnosis. In this report we compare two different OCT systems: an $890 \mathrm{~nm}$ spectrometer-based OCT system with $2.5 \mu \mathrm{m}$ axial resolution and a $1300 \mathrm{~nm}$ swept-source OCT system with $7.5 \mu \mathrm{m}$ axial resolution to determine the effect of these different OCT parameters on the endogenous backscatter contrast of dysplastic/malignant oral mucosa models relative to normal mucosa models. Tissueengineered oral mucosa models constructed with a dysplastic cell line (DOK), a malignant cell line (Cal27) and normal cell were imaged with both of these OCT platforms and comparisons made with regard to apparent epithelial thickness and the visibility of the epithelium relative to the underlying stroma. For the Cal27's, hematoxylin and eosin staining confirmed the formation of a keratinized layer superficial to a thickened layer of viable cells on top of the stroma. The keratinized layer presented as a hyperreflective thickened layer superficial to a darker region on both OCT platforms. The keratinized layer caused a steep fall in signal at $890 \mathrm{~nm}$, making it difficult to visualise underlying structures, whereas $1300 \mathrm{~nm}$ OCT clearly visualized both the epithelial cells and the stroma lying beneath. For the DOK cells, hematoxylin and eosin staining confirmed the formation of an epithelial layer frequently presenting an abnormal morphology especially at the epidermal/stromal junction, with features such as infiltrating, bulbous rete pegs. These were more clearly visualized under $890 \mathrm{~nm}$ OCT.

These observations show that $890 \mathrm{~nm}$ OCT retains some of its known advantages of higher contrast between anatomical tissue layers when used to observe dysplastic and malignant 3D oral mucosa constructs. However $1300 \mathrm{~nm}$ OCT is confirmed to possess a greater ability to image the full thickness of the model epithelia and in particular it is more suited to imaging through the keratinized layer.

Key words: epithelium; oral mucosa; optical coherence tomography; tissue engineering.

Introduction. The early detection of dysplasia and invasive cancer in epithelial tissue remains high priority in the medical area. Over $80 \%$ of all primary tumours are believed to arise in the epithelial tissue before metastasising to other locations via the blood or lymphatic systems. The infiltration of epithelial cancer cells through the basement membrane and into the vascularized stroma thus represents a key diagnostic landmark which, if detected sufficiently early, could greatly influence the subsequent therapeutic pathway.

Photonic imaging techniques are an ideal tool with which to attempt such early detection because of the speed, cost-effectiveness, spatial resolution and molecular sensitivity offered. Biophotonic techniques can potentially be developed into tools for non-invasively determining tumour resection margins and for guiding the site of biopsy sampling to reduce false negatives when biopsying diffuse disease.

The imaging depth of optical and near-infrared is limited by intense light scattering penetration, however this is not a major disadvantage for epithelial cancer detection as the epithelium is generally just a few hundred microns thick.

Corresponding author: Stephen J. Matcher, e-mail: S.J.Matcher@sheffield.ac.uk 
Endoscopic confocal microscopy can potentially detect changes in cell nuclear morphology, a recognised marker for dysplastic change [1, 2], non-invasively. Compared with confocal microscopy optical coherence tomography (OCT) has a lower spatial resolution but has a much higher voxel acquisition rate [3]. It is thus potentially better suited to establishing the gross morphology and extent of a tumour and for example OCT shown potential in detecting basal cell carcinoma nests [4]. However it is generally found that it lacks the spatial resolution to directly measure changes in nuclear morphology and consequently it lacks sufficient contrast between normal and abnormal tissues to provide a robust alternative to conventional histology.

It is accepted that the OCT imaging wavelength and source bandwidth are strong determinants of the final image contrast. Sources with centre wavelength below $1 \mu \mathrm{m}$ are available with bandwidths in excess of $150 \mathrm{~nm}$, giving them a decisive advantage in terms of achievable axial resolution. $1300 \mathrm{~nm}$ systems on the other hand offer better depth penetration due to the consistently lower attenuation coefficients at the longer wavelength. It is therefore important to assess the image quality offered by these two wavelength bands in particular situations.

An early report into the use of dual-band Fourier domain OCT for imaging the skin used a supercontinuum source to simultaneously image two OCT spectrometers, one operating at $800 \mathrm{~nm}$ and the other at $1250 \mathrm{~nm}$ [5]. Images collected on the palmar skin at the distal end of the little finger showed greater depth penetration and generally higher backscatter levels from the stratum granulosum for the $1250 \mathrm{~nm}$ image. Backscatter from the stratum corneum was higher when measured by the $800 \mathrm{~nm}$ channel, with the stratum granulosum appearing somewhat less distinct.

Alex et al. [6] systematically evaluated the imaging performance of OCT platforms operating at 800, 1060 and $1300 \mathrm{~nm}$ when applied to in vivo normal human skin. A titanium-sapphire femtosecond laser source with $140 \mathrm{~nm}$ bandwidth offered $3 \mu \mathrm{m}$ axial resolution and $12 \mu \mathrm{m}$ lateral resolution and this displayed improved contrast between epithelial layers and dermis when compared with a $1320 \mathrm{~nm}$ system based on SLD's and offering 7 micron axial resolution and $15 \mu \mathrm{m}$ lateral resolution. The $1300 \mathrm{~nm}$ system however offered markedly better depth penetration.

In this study we compare the OCT presentation of three tissue-engineered oral mucosa models using an $890 \mathrm{~nm}$ SLD-based spectrometer system and a commercial 4-beam swept-source system operating at $1300 \mathrm{~nm}$. The OCT morphology and contrast are compared with hematoxylin and eosin stained histology.

Materials and Methods

$890 \mathrm{~nm}$ OCT system. The $890 \mathrm{~nm}$ system used for imaging oral mucosa models is a custom-built ultrahigh resolution spectral domain optical coherence tomography system. A dual super luminescent diode source (Broadlighter D890-HP, Superlum, Ireland) emits a central wavelength of $890 \mathrm{~nm}$ and a bandwidth of $150 \mathrm{~nm}$ into the single mode fibre based system. The emitted beam is split into the reference and sample beams using a 50/50 coupler (Gould Fiber Optics, USA) fabricated from Nufern 630-HP fiber (USA). The sample beam is deflected by an
$X-Y$ galvo pair and focused by a telecentric OCT scan lens (LSM02-BB, Thorlabs, Inc., USA) with an effective focal length of $18 \mathrm{~mm}$. The reference arm consists of a fixed plane mirror, an adjustable neutral density filter and a dispersion compensator (LSM02DC, Thorlabs, Inc., USA) to partially compensate for the glass focusing lens in the sample arm.

The recombined sample and reference beam are detected by a spectrometer constructed in-house. The output beam is incident on an achromatic doublet lens (AC254-075-B-ML, Thorlabs, Inc., USA), which then focuses the beam onto a $1200 \mathrm{lp} / \mathrm{mm}$ diffraction grating (Wasatch Photonics, Inc., USA). The dispersed beam is refocused onto a line scan CCD (Aviiva, EV71YEM1GE2014-BA9, e2v, UK) via a lens doublet with $200 \mathrm{~mm}$ focal length each. A-scans (depth profiles) were acquired at a rate of $20 \mathrm{kHz}$ and $\mathrm{B}$-scans were assembled consisting of $1000 \mathrm{~A}$-scans. After subtraction of the d.c. component, fringes were resampled using a technique based on detecting the fringe zero-crossings and using the interpolated pixel positions of these to build a nonlinear interpolation table between pixel value and k. Pixels outside the interference spectrum are zeroed. Following these steps the system has a measured axial resolution in air of $2.6 \mu \mathrm{m}$. The lateral resolution was measured using the USAF resolution target (Edmund Optics, USA) and found to be $8 \mu \mathrm{m}$. With a plane mirror as the sample a sensitivity of $93 \mathrm{~dB}$ was achieved with a $6 \mathrm{~dB} / \mathrm{mm}$ roll off.

$1300 \mathrm{~nm}$ OCT system. A 4-beam swept source Fourier domain OCT system (EX1301, Michelson Diagnostics Ltd., UK) with Santec, Inc., HSL-2000-10 swept laser (Japan) (center wavelength of approximately $1300 \mathrm{~nm}$ and bandwidth of approximately $150 \mathrm{~nm}$ ) was used, giving a twodimensional cross-sectional visualization of micro-structural morphology at 10,000 A-scans per second. B-scans comprising $952 \mathrm{~A}$-scans are acquired along $4 \mathrm{~mm}$ in lateral direction, at a rate of 5 frames per second. Axial resolution in air is better than $7.5 \mu \mathrm{m}$ and lateral resolution at best focus approaches $7.5 \mu \mathrm{m}$ also. Software stitching combines the region of best focus from four independently focussed beams together to provide a composite image with $\times 2$ better mean lateral resolution than a single beam interrogating the same axial range [7]. The system has demonstrated clinical value in the identification of malignant and potentially malignant disorders of the oral mucosa, achieving a sensitivity of $85 \%$ and specificity of $78 \%$ when used to image biopsy samples in an immediate ex vivo study [8].

Tissue-engineered normal and dysplastic oral mucosa models. A 3D model of healthy human oral buccal mucosa has been developed within our institute, which consists of an epithelium with underlying connective tissue component. This tissue model has been developed in the MacNeil laboratory and has been developed for clinical applications [9]. This tissue-engineered oral mucosa (TEOM) has been successfully used in the clinic to replace scarred tissue in the urethra with three year follow up [10]. Models of dysplasia and carcinoma in situ are generated by replacing the normal oral keratinocytes with dysplastic or malignant cell lines.

All cell culture work was carried out in class II laminar flow hoods (Walker Safety Cabinets, UK). Laboratory 
plastic wear for cell culture was purchased from Costar, UK. All cells were incubated at $37^{\circ} \mathrm{C}$ in humidified incubators containing $5 \% \mathrm{CO}_{2}$.

The TEOM were cultured on an acellular skin scaffold processed from glycerol treated cadaveric skin purchased from Euroskin Bank (Beverwijk, the Netherlands). The skin stored in glycerol was stored in at $4^{\circ} \mathrm{C}$ until needed. It was then washed in sterile PBS extensively to remove the glycerol. Following washes with sterile PBS the skin was incubated in $1 \mathrm{M}$ sodium chloride for 24 hours at $37^{\circ} \mathrm{C}$. The epidermis from the skin was peeled off and DED (deepithelialised dermis) was used as scaffold for TEOM. The DED was stored at $4^{\circ} \mathrm{C}$ in medium until further use.

The DED was cut into $1.5 \times 1.5 \mathrm{~cm}$ squares and placed into a 6 well plate. Chamfered rings are placed on top of the DED and weighted down in order to ensure a liquid tight seal was created between the ring and the DED. Normal oral keratinocytes (NOK) and normal oral fibroblasts (NOF) were isolated from waste tissue collected during routine oral surgery (Ethics reference 09/H1308/66) as described by Colley et al. [11]. They were then seeded in the wells of the rings in a $3: 1$ ratio i.e. 300,000 oral keratinocytes and 100,000 oral fibroblasts in Green's media. On day 2 post cells seeding the composites were raised to air liquid interface (ALI) on a stainless steel grid and cultured for 7 , 14 and 21 days.

Two different cell lines were used to produce different abnormal mucosal models: the Cal27 oral squamous cell carcinoma (OSCC) cell line and the DOK preinvasive cell line. These were produced in the same way as the normal mucosa, except that only 250,000 cells of each type were added to 100,000 NOF. These models were grown in media corresponding to their cell line. All models were cultured for 7,14 , and 21 days. Cal27 is an oral squamous cell carcinoma human cell line which was isolated from the tongue and cultured in DMEM with 10\% FCS. The Cal27 cells were purchased from ATCC (Manassas, VA, USA). DOK (ECACC 94122104) is a dysplastic oral keratinocyte cell line isolated from a lesion in the tongue. The lesion was adjacent to an OSCC and showed mild to moderate dysplasia. DOK cells were obtained from the European Collection of Cell Culture (ECACC) via Sigma Aldrich (Dorset, UK). Cells were cultured in DMEM with 10\% FCS, supplemented with hydrocortisone ( $5 \mu \mathrm{g}$ per $\mathrm{mL})$.

\section{Results}

Oral mucosa models - histological assessment. In establishing these models we have determined that the length of time the models are cultured at air liquid interface affects the thickness of the epithelium and degree of stratification [11, 12]. Culturing the TEOM at an air liquid interface produces a stratified epithelium after 7 days in culture (Figure 1).

By 21 days in culture the epithelium is up to 10 cells thick and shows clear signs of organisation and differentiation. Over time in culture the model becomes more organised and the epithelium becomes thicker. When the hematoxylin and eosin stained histology sections are compared against healthy oral mucosa biopsy (Figure 2), similarities between the tissue-engineered model and the human biopsy are apparent. Although the TEOM model has a thinner epithelium compared to the biopsy the differentiation pattern which is observed in vivo can also be seen in our tissue-
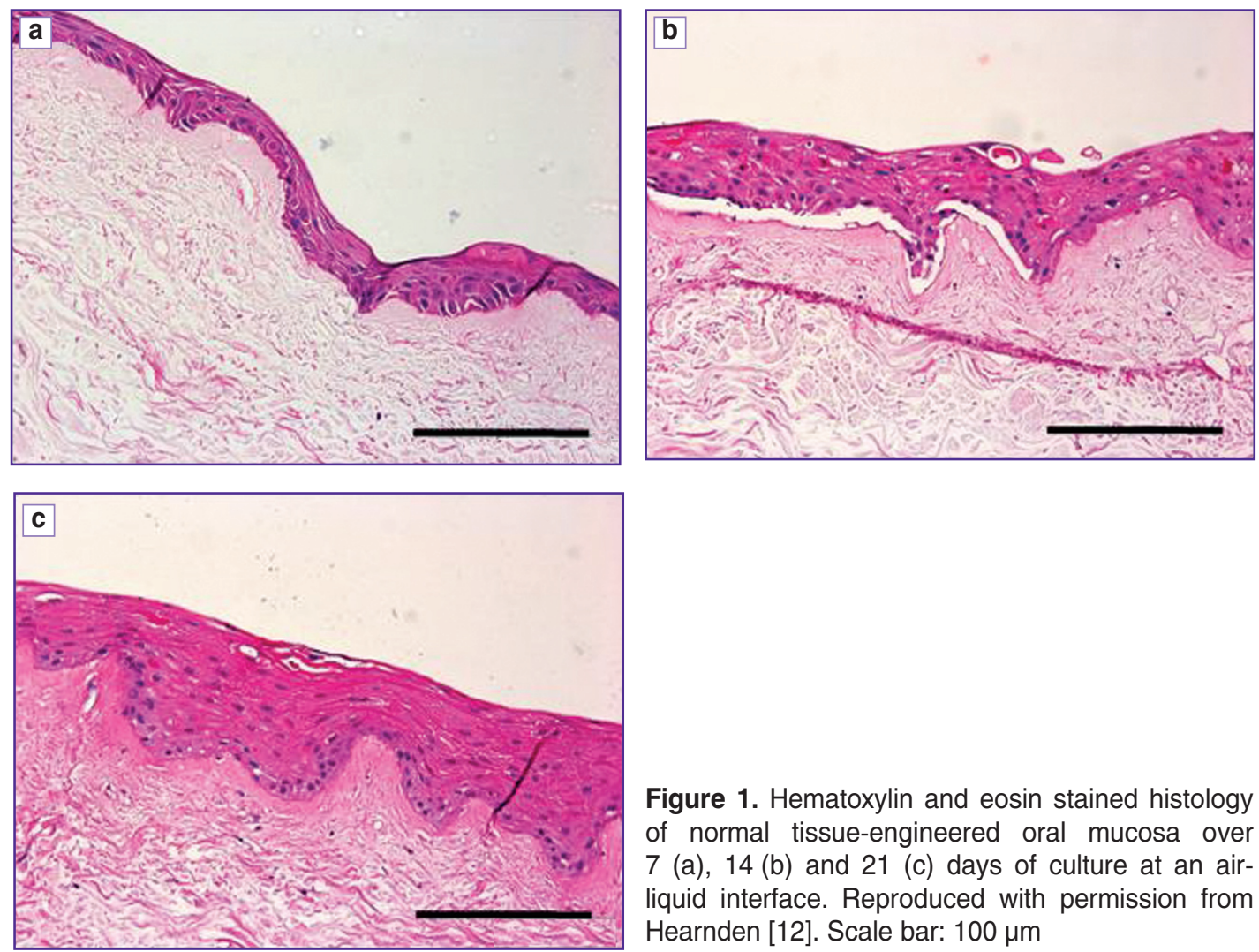

Figure 1. Hematoxylin and eosin stained histology of normal tissue-engineered oral mucosa over 7 (a), 14 (b) and 21 (c) days of culture at an airliquid interface. Reproduced with permission from Hearnden [12]. Scale bar: $100 \mu \mathrm{m}$ 

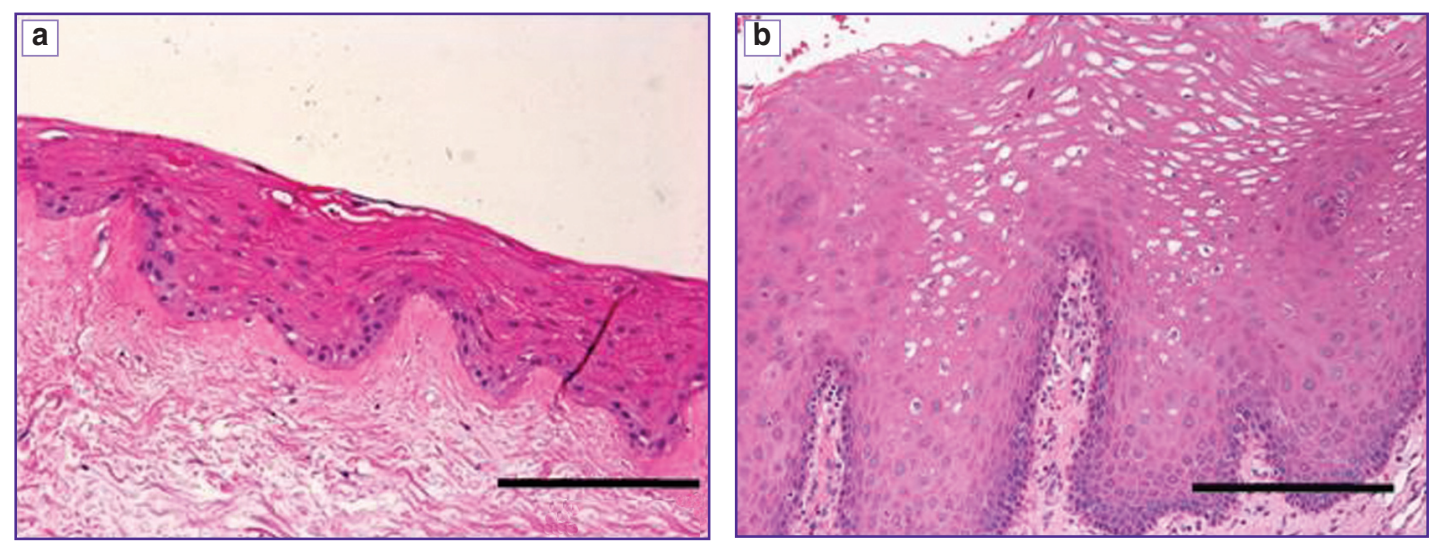

Figure 2. Hematoxylin and eosin stained histological comparison of the normal TEOM (a) with an in vivo biopsy of healthy oral mucosa (b). Reproduced from Hearnden [12] with permission. Scale bar: $100 \mu \mathrm{m}$

engineered model. The basal cells are densely packed with a high number of nuclei in the basal region of the epithelium. Superficially the basal and the supra-basal cells are larger and the nuclei are more dispersed as the cells are beginning to differentiate. The most superficial layer contains cells which are flattened with very few nuclei. These cells are terminally differentiated and have produced a smooth flat surface to the epithelium. Both the human biopsy and the TEOM have rete ridges, the characteristic rounded appearance of the epithelial connective tissue junction. The cells of the epithelium have differentiated in strata (rather than individual cells), a sign that intracellular signalling has been involved in the evolution of the epithelium.

Figure $3(\mathrm{a})-(\mathrm{f})$ show the development of the 3D tissueengineered models resulting from DOK cells cultured on an acellular skin scaffold. After 7 days in culture at ALI the DOK cells form a multi-layer epithelium of between 2-10 cells thick. There are areas which show signs of cell death and the cells have not differentiated into distinct strata (as was seen with the normal cells). After 14 days in culture the epithelium is thicker than at 7 days and the strata of the epithelium are beginning to show signs of organising themselves. A few of the superficial cells are becoming flattened and the cells adjacent to the connective tissue are in some cases organising into a pattern seen in normal epithelia. There are signs of individual cells producing keratin rather than strata of cells (a sign of dysplasia) along with nuclear pleomorphism throughout the epithelium (an indicator of severe dysplasia or carcinoma in situ). Significant cytological atypia is seen in the basal cell which is a feature commonly seen in carcinomas (highlighted region of Figure $3(d)$ ). By 21 days in culture the epithelium has a severely dysplastic, carcinoma in situ appearance with cytological and architectural abnormalities throughout the epithelium. These include: drop shaped rete peg (bulbous projection into the connective tissue) and keratin pearls (circular formations of keratin, stained pink by hematoxylin and eosin) in the middle region of the epithelium. There are islands of cells within the stroma which have the appearance of epithelial cell invasion. These cells have a different appearance to fibroblasts in the connective tissue as fibroblasts do not appear to cluster in group as the cells seen in these models do and there is hematoxylin staining around the cells (not seen for the fibroblasts).

The Cal27 cells produce models which are most comparable to the appearance of clinically occurring dysplasia and carcinoma in situ (Figure 4). After 7 days in culture the epithelium is found to be well differentiated with a well-defined basal cell layer. The differentiation occurs in regions and strata of the epithelium rather than in individual cells indicating some cell-cell interactions. Islands of epithelial cell are found within the connective tissue suggesting invasion of the OSCC into the connective tissue. The models cultured for 14 days appear highly keratinised on the surface. This high level of keratinisation is also commonly seen on the surface of dysplastic tissues clinically. Figure 4 (c) shows the organised differentiation remaining in areas however, in other places this is disrupted by the extensive keratinisation.

After 21 days in culture the appearance of the histology is less like dysplastic lesions seen clinically as cells begin to grown over the top of the keratinised areas and the differentiation is mostly lost. This model has the appearance of a squamous cell carcinoma in situ with some signs of invasion as early as 7 days at ALI.

Oral mucosa models - OCT presentation at 890 and $1300 \mathrm{~nm}$. Figure 5 shows OCT B-scans collected on the three oral mucosa models at day 21 .

It is clear that the $890 \mathrm{~nm}$ images generally present darker and with a greater roll-off in signal strength with depth than the $1300 \mathrm{~nm}$ images. In part this will be due to different system parameters, however it is clear from comparing image (c) and image (f) (See Figure 5) that there is also greater contrast between the tissue-engineered epithelium and the DED when imaging at $890 \mathrm{~nm}$ and with 2.5 micron axial resolution. This translates into an improved ability to infer the epithelial thickness and, for the DOK model in image (c) (See Figure 5), a clear ability to resolve morphological abnormalities such as infiltrating, drop shaped rete pegs similar to those shown by histology (See Figure $3(\mathrm{f})$ ).

For the Cal27 models there is a clear inversion of the usual pattern of contrast for OCT images of epithelium, with the most superficial band appearing brighter than 


\section{NOVEL STUDIES}
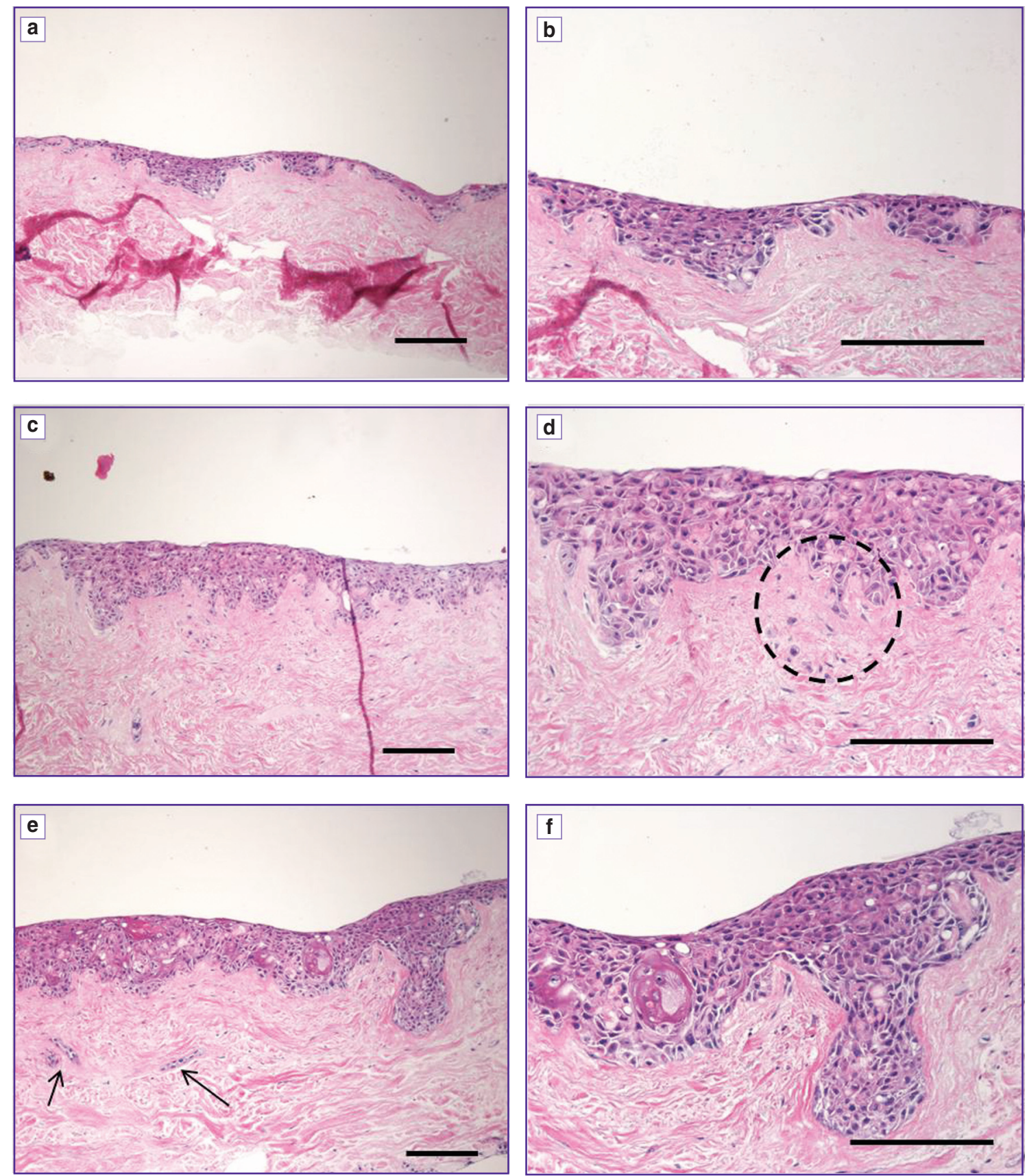

Figure 3. Hematoxylin and eosin stained histological sections of the DOK cell line cultured in three dimensions on DED. Models were grown at an air liquid interface for 7 days $\times 10$ magnification (a) and $\times 20$ magnification (b); 14 days $\times 10$ magnification (c) and $\times 20$ magnification (d) or 21 days $\times 10$ magnification (e) and $\times 20$ magnification (f). Highlighted circle (d) shows invading cells. Arrows (e) show invaded tumour islands. Scale bar: $200 \mu \mathrm{m}(\mathrm{n}=3)$. Reproduced from Hearnden [12] with permission

the underlying layers. At $890 \mathrm{~nm}$ this superficial layer is considerably brighter than the corresponding layer in the normal model and displays a sharp, irregular lower border indicative of strong contrast between this layer and the underlying material. Even fainter signal is visible below this underlying layer, particularly towards the image centre. Based on our previous experience with OCT imaging of tissue-engineered skin [13], we consider that a plausible interpretation of the Cal27 images is that the brighter superficial region represents keratinized tissue overlying the metabolically active dysplastic epithelial cells (which generally present more darkly than stroma in OCT images). Such keratinization is a late-stage feature of the Cal27 models (See Figure 4 (f)) as well as being a common clinical feature of dysplastic oral lesions. There are small dark infiltrations at the surface of model (especially clear on the $890 \mathrm{~nm}$ image), which may be areas of nascent epithelial cell overgrowth. The weak signal seen deep in the Cal27 
model may be the remnants of signal from the underlying DED, which is now deep below both the keratinized superficial layer and a thickened layer of epithelial cells.

Figure 6 shows a volumetric flythrough of the same area of a DOK oral mucosa construct, imaged by ultrahigh resolution OCT (upper) and multi-beam OCT (lower). The DOK construct was imaged at day 21. 573 slices were acquired, each $4 \mathrm{~mm}$ wide and with 5 micron slice spacing. Data was collected sequentially on the two systems. Fiducial markers were placed on the epithelia surface of the construct, to facilitate the approximate registration of the two data sets. It is clear that the $1300 \mathrm{~nm}$ image shows more signal from deeper regions of the stroma whereas the $890 \mathrm{~nm}$ image more clearly demarcates the epithelial thickness, epidermal/stromal junction and epidermal infiltrations into the stroma.

Conclusions. OCT images collected at 1300 and $890 \mathrm{~nm}$ displayed different levels of contrast between the epithelium and stroma in both the normal and dysplastic models. There is a general trend for the $890 \mathrm{~nm}$ images to show greater
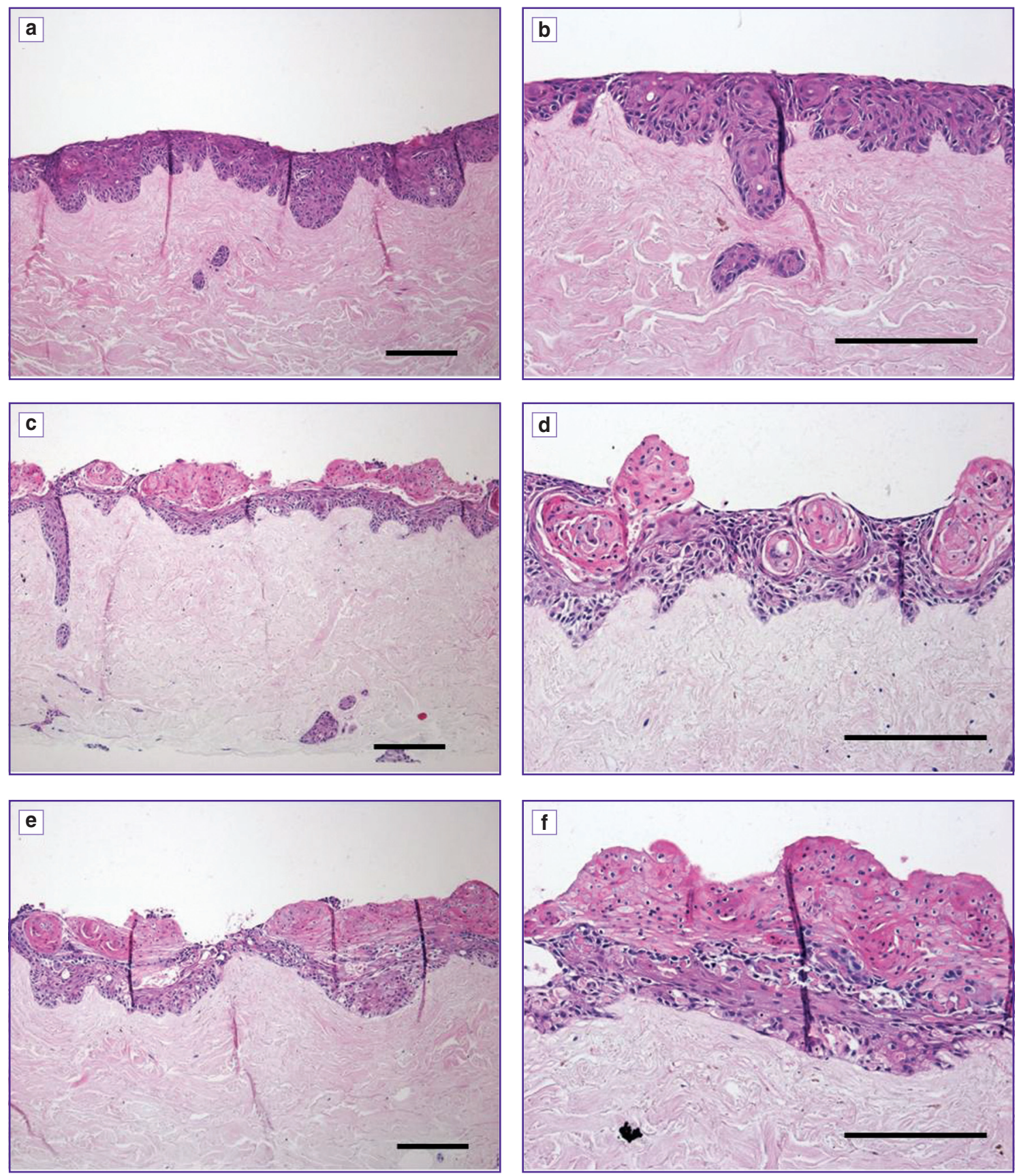

Figure 4. Hematoxylin and eosin stained histological sections of the Cal27 cell line cultured in three dimensions on DED. Models were grown at an air liquid interface for 7 days $\times 10$ magnification (a) and $\times 20$ magnification (b); 14 days $\times 10$ magnification (c) and $\times 20$ magnification (d) or 21 days $\times 10$ magnification (e) and $\times 20$ magnification ( $f$ ). Scale bar: $200 \mu \mathrm{m}(\mathrm{n}=3)$. Reproduced from Hearnden [12] with permission 


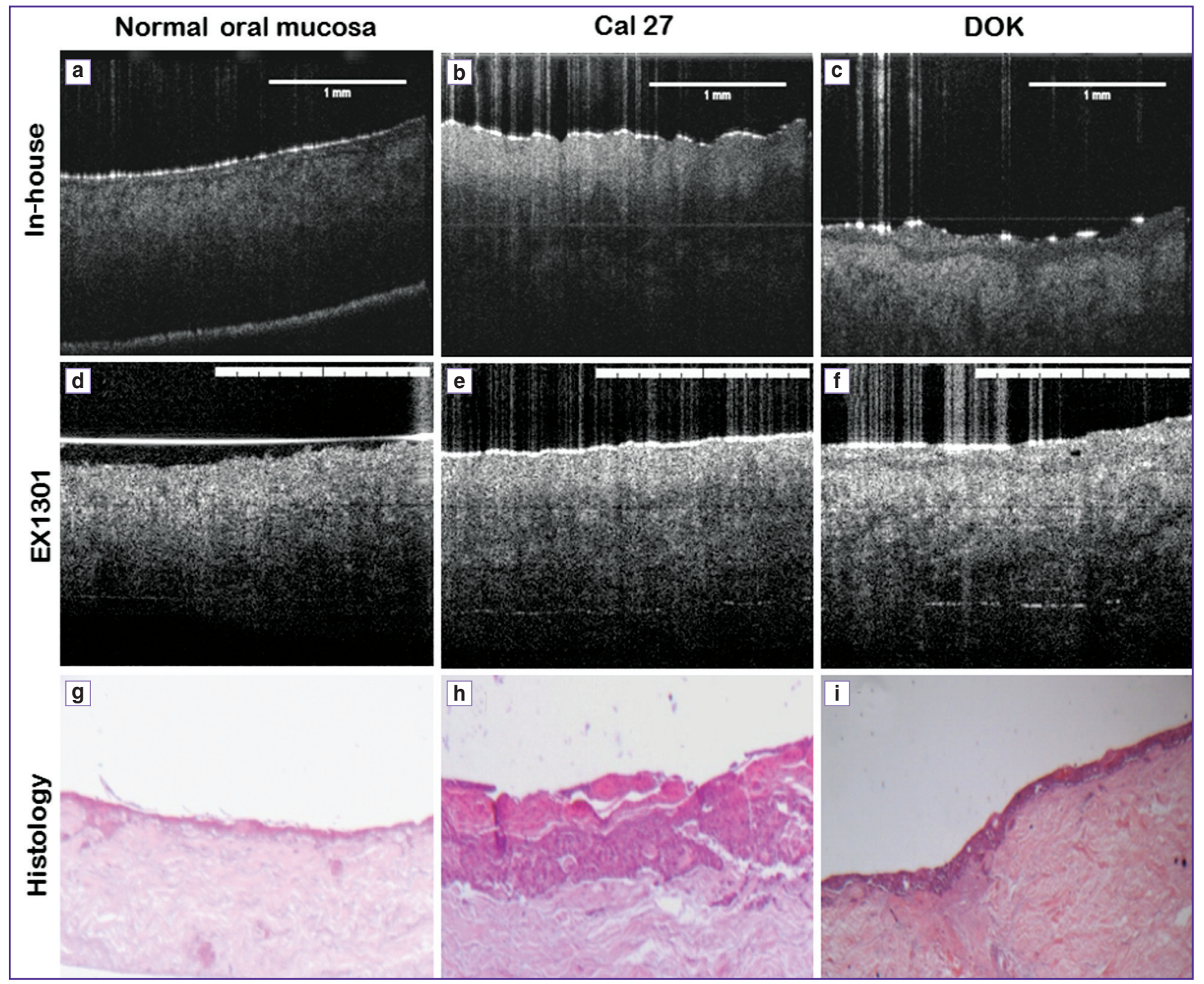

Figure 5. Three oral mucosa models imaged using $890 \mathrm{~nm}$ (upper row) and $1300 \mathrm{~nm}$ OCT (middle row). Scale bar: $1 \mathrm{~mm}$. Contemporary histology shown on the lower row

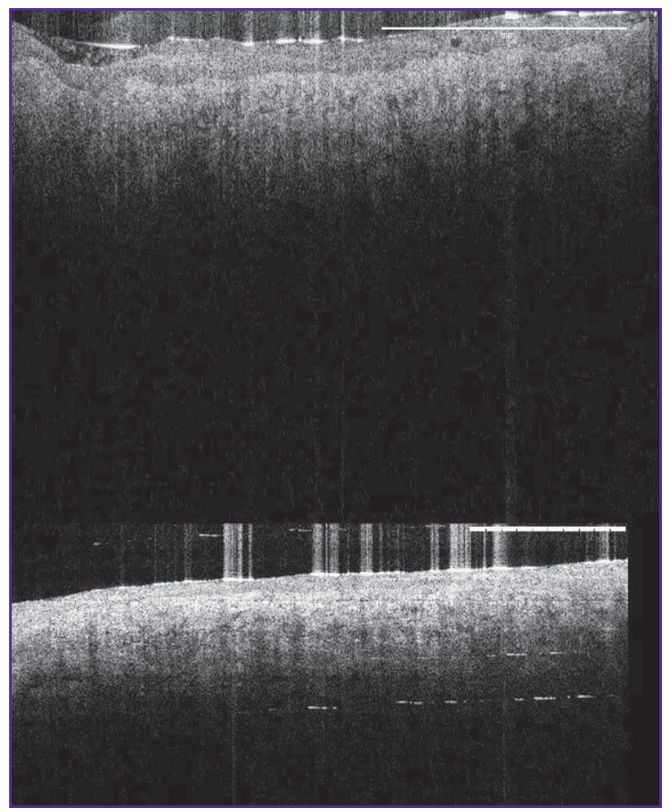

Figure 6. Single frame from a volumetric flythrough of a DOK model imaged at 21 days using the $890 \mathrm{~nm}$ OCT system (upper) and the $1300 \mathrm{~nm}$ OCT (lower). See also Media. Scale bar: $1 \mathrm{~mm}$ 
contrast between the different layers, herein interpreted as epithelium vs stroma (normal and DOK models) and keratinized layer vs epithelium vs stroma (Cal27 $\mathrm{m}$ odel). The $1300 \mathrm{~nm}$ images consistently show better depth penetration however, and a more uniform contrast across the imaging depth, in agreement with studies on in vivo skin. Dual wavelength imaging may therefore be a fruitful avenue to explore for improved imaging of oral lesions, with the $890 \mathrm{~nm}$ channel revealing morphological irregularities in the epithelium with greater sensitivity and with the $1300 \mathrm{~nm}$ channel providing better sensitivity to deeper structures such as the vasculature [14].

Study Funding. EPSRC grants EP/EI018328, EP/ F020422.

Conflict of Interest. The authors have no conflict of interests to disclose.

\section{References}

1. Collier T., Lacy A., Richards-Kortum R., Malpica A., Follen M. Near real-time confocal microscopy of amelanotic tissue: detection of dysplasia in ex vivo cervical tissue. Acad Radiol 2002; 9(5): 504-512, http://dx.doi.org/10.1016/s10766332(03)80326-4.

2. Hoffman A., Goetz M., Vieth M., Galle P.R., Neurath M.F., Kiesslich R. Confocal laser endomicroscopy: technical status and current indications. Endoscopy 2006; 38(12): 1275-1283, http://dx.doi.org/10.1055/s-2006-944813.

3. Zysk A.M., Nguyen F.T., Oldenburg A.L., Marks D.L., Boppart S.A. Optical coherence tomography: a review of clinical development from bench to bedside. J Biomed Opt 2007; 12(5): 051403, http://dx.doi.org/10.1117/1.2793736.

4. Coleman A.J., Richardson T.J., Orchard G., Uddin A., Choi M.J., Lacy K.E. Histological correlates of optical coherence tomography in non-melanoma skin cancer. Skin Res Technol 2013; 19(1): e10-e19, http://dx.doi.org/10.1111/ j.1600-0846.2012.00626.x.

5. Cimalla P., Walther J., Mehner M., Cuevas M., Koch E. Simultaneous dual-band optical coherence tomography in the spectral domain for high resolution in vivo imaging. Opt
Express 2009; 17(22): 19486-19500, http://dx.doi.org/10.1364/ OE.17.019486.

6. Alex A., Povazay B., Hofer B., Popov S., Glittenberg C., Binder S., Drexler W. Multispectral in vivo three-dimensional optical coherence tomography of human skin. $J$ Biomed Opt 2010; 15(2): 026025, http://dx.doi.org/10.1117/1.3400665.

7. Holmes J. In vivo real-time optical coherence tomography imaging of Drosophila for cardiovascular research. Nat Methods 2009; 6(10): III-IV.

8. Hamdoon Z., Jerjes W., Upile T., McKenzie G., Jay A., Hopper C. Optical coherence tomography in the assessment of suspicious oral lesions: an immediate ex vivo study. Photodiagnosis Photodyn Ther 2013; 10(1): 17-27, http:// dx.doi.org/10.1016/j.pdpdt.2012.07.005.

9. Bhargava S., Chapple C.R., Bullock A.J., Layton C., MacNeil S. Tissue-engineered buccal mucosa for substitution urethroplasty. BJU Intern 2004; 93(6): 807-811, http://dx.doi. org/10.1111/j.1464-410X.2003.04723.x.

10. Bhargava S., Patterson J.M., Inman R.D., MacNeil S., Chapple C.R. Tissue-engineered buccal mucosa urethroplasty — clinical outcomes. Eur Urol 2008; 53(6): 12631271, http://dx.doi.org/10.1016/j.eururo.2008.01.061.

11. Colley H.E., Hearnden V., Jones A.V., Weinreb P.H., Violette S.M., Macneil S., Thornhill M.H., Murdoch C. Development of tissue-engineered models of oral dysplasia and early invasive oral squamous cell carcinoma. $\mathrm{Br}$ J Cancer 2011; 105(10): 1582-1592, http://dx.doi.org/10.1038/ bjc.2011.403.

12. Hearnden V. Developing tissue engineered models of oral mucosa and oral cancer to study novel therapeutic and diagnostic techniques. PhD Thesis. University of Sheffield; 2011.

13. Smith L.E., Hearnden V., Lu Z., Smallwood R., Hunter K.D., Matcher S.J., Thornhill M.H., Murdoch C., MacNeil S. Evaluating the use of optical coherence tomography for the detection of epithelial cancers in vitro. J Biomed Opt 2011; 16(11): 116015, http://dx.doi.org/10.1117/1.3652708.

14. Enfield J., Jonathan E., Leahy M. In vivo imaging of the microcirculation of the volar forearm using correlation mapping optical coherence tomography (cmOCT). Biomed Opt Express 2011; 2(5): 1184-1193, http://dx.doi.org/10.1364/ BOE.2.001184. 